\title{
Effects of synbiotic supplement on LPS level in Thai smokers: A preliminary study
}

\author{
Parama Pratummas ${ }^{1}$, Vachrintr Sirisapsombat ${ }^{2}$, Phuttharaksa Phumcharoen ${ }^{3}$, \\ Thamthiwat Nararatwanchai ${ }^{4}$, Chaiyavat Chaiyasut ${ }^{5}$, Sasithorn Sirilun ${ }^{6}$, \\ Phakkharawat Sittiprapaporn ${ }^{7}$
}

${ }^{1,2,3}$ Doctoral Student, Brain Science and Engineering Innovation Research Group, School of Anti-Aging and Regenerative Medicine, Mae Fah Luang University, Bangkok, Thailand, and Department of Anti-Aging Medicine, School of Anti-Aging and Regenerative Medicine, Mae Fah Luang University, Bangkok, Thailand, ${ }^{4}$ Professor, Department of Anti-Aging Medicine, School of Anti-Aging and Regenerative Medicine, Mae Fah Luang University, Bangkok, Thailand, ${ }^{5}$ Assistant Professor and Head, ${ }^{6}$ Assistant Professor, Innovation Center for Holistic Health, Nutraceuticals and Cosmeceuticals, Faculty of Pharmacy, Chiang Mai University, Chiang Mai, Thailand, ${ }^{7}$ Assistant Professor and Head, Brain Science and Engineering Innovation Research Group, School of Anti-Aging and Regenerative Medicine, Mae Fah Luang University, Bangkok, Thailand, and Department of Anti-Aging Science, School of Anti-Aging and Regenerative Medicine, Mae Fah Luang University, Bangkok, Thailand

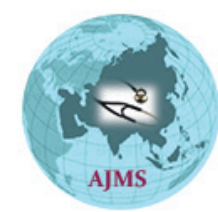

\section{A B S T R A C T}

Background: The impacts of smoking on the intestinal microbiome piece and arranged assortment have been appeared in different arranged controlled and observational clinical trials in individuals. Synbiotics are utilized not fair for the progressed survival of beneficial microorganisms included to food, however in expansion for the prompting of the duplication of specific nearby bacterial strains display within the gastrointestinal tract. Aims and Objective: This research proposed the impact of synbiotic supplement on gut-brain axis in Thai smokers in the realm of gut-related biochemical parameters and cognition. Materials and Methods: We directed a single gathering, pre-and post-test studies; 20 participants lived in Mahasarakham, Fagerstrom Test for Nicotine Dependence at 4 or above, and no history of cerebrovascular diseases, brain and abdominal surgery, and not taking hormones or medications impacted brainwaves or utilizing antibiotics inside about fourteen days prior. Participants were allocated to synbiotic supplement containing 3 kinds of prebiotics and 7 types of probiotics prior to sleep every day for about two months. Results: Results were estimated in gut-related biochemical parameters change and alternation in cognition. The present outcomes have indicated essentially decline in the scores of Fagerstrom Test for Nicotine Dependence and also the scores of craving for smoke after intervention. In additions, lipopolysaccharide (LPS) in plasma significantly decreased after the intervention. Conclusion: Further researches are obligatory to lead in finding a propensity to improve in gut-related biochemical parameter and cognition after admission of synbiotic supplement.
Access this article online

Website:

http://nepjol.info/index.php/AJMS

DOI: 10.3126/ajms.v11i1.26495

E-ISSN: 2091-0576

P-ISSN: $2467-9100$

Key words: Synbiotics; Gut Microbiota; Gut-Brain Axis; Cigarette Smoker; Cognition

\section{INTRODUCTION}

The tobacco epidemic is one of the most prominent common well-being perils the world has ever gone up against, butchering in overabundance of 7 million people each year. In overabundance of 6 million of those losses are the result of coordinate tobacco utilize whereas around 890,000 are the eventual outcome of non-smokers being displayed to second-hand smoke. ${ }^{1}$ In Thailand, the observation of smoking propensity led in $2014 \mathrm{had}$ demonstrated that $20.7 \%$ of 54.8 million individuals matured over 15 years of age were smoking, considered $18.2 \%$ as standard smokers and $2.5 \%$ as periodic smokers. ${ }^{2}$ Tobacco smoke is a complex, dynamic and responsive blend containing an expected 5,000 synthetics. ${ }^{3-5}$ The most broadly recognized degenerative diseases from smoking are

Address for Correspondence:

Dr. Phakkharawat Sittiprapaporn, Assistant Professor and Head, Brain Science and Engineering Innovation Research Group School of Anti-

Aging and Regenerative Medicine, Mae Fah Luang University Bangkok 10110, Thailand. Phone: +662 6644361. E-mail: wichian.s@mfu.ac.th (c) Copyright AJMS 
cardiovascular diseases, pneumonic illnesses, and malignant diseases. Smoking also increases perinatal issues, for case, low birth weight and unsuccessful labor. The foremost broadly recognized mental comorbidities are liquor/ substance, burdensome, bipolar, uneasiness, character, and attention-deficit/hyperactivity disorders. In individuals with current tobacco utilize issue, the commonness of current alcohol, and medicine, apprehension, burdensome, bipolar, and character issue ranges from $22 \%$ to $32 \%$. Nicotinedependent smokers are 2.7-8.1 events bound to have these disarranges than nondependent smokers, never-smokers, or ex-smokers. ${ }^{6}$

In various smokers, the foremost concerning parts of ceasing are the decided slants or needs to smoke a cigarette. Cravings will in common diminishment in quality and repeat with longer restraint period, all things considered a minority of ex-smokers still report compelling impulses a half year within the wake of ceasing. ${ }^{7}$ Empirical studies have illustrated that cravings - a crucial component of tobacco utilize issue within the DSM-V - are one of the foremost relentless markers of backslide in ex-clients, and suitably their diminished is a basic objective of conclusion treatment in smokers. ${ }^{8}$ Longing for is routinely characterized as a unique experience of requiring to utilize a medicine, and in this way arranging oneself toward pharmaceutical taking. Ancient fashion molding models stipulate that wants are circumstance express and innovative, to such a degree that they can be enacted by boosts as of late associated with medicine utilize, and reestablished an awfully long time after restriction. ${ }^{9}$ Tiffany and Conklin have supplemented this model by contending that craving could be a multifaceted construct that incorporates diverse mental strategies, counting memory of past pharmaceutical taking and expectation of resulting utilize. As shown by them, drug-taking behaviors may happen without longing for in light of the truth that they ended up automatized within the advance arrange to dependence. The work of needing would in this way contain of subjective methods that can fuel or deflect the execution of automatized pharmaceutical utilize penchants. ${ }^{10}$

The impacts of smoking on the intestinal microbiome piece and arranged assortment have been appeared in different arranged controlled and observational clinical trials in individuals. ${ }^{11-17}$ Synbiotics are utilized not fair for the progressed survival of beneficial microorganisms included to food, however in expansion for the prompting of the duplication of specific nearby bacterial strains display within the gastrointestinal tract. ${ }^{18}$ Concerning their therapeutic efficacy, the alluring properties of synbiotics join antibacterial, anticancerogenic, and unfriendly to unfavorably vulnerable impacts. They likewise neutralize decay shapes within the stomach related tract and neutralize constipation and diarrhea. For reasons obscure, synbiotics could be exceptionally efficient within the evasion of osteoporosis, diminish of blood fat and sugar levels, rule of the immunological system, and treatment of brain issue related with abnormal hepatic capacity. ${ }^{19}$

There are numerous reports demonstrated that smokers have gut dysbiosis in them and also the inflammation state. Inflammation can be caused in different ways, and lipopolysaccharide (LPS) is an important tool for this function. LPS is a protein that is found in the outer membrane of Gram-negative bacteria. Its main target is the toll-like receptor (TLR) 4, although it is known to function on other receptors. ${ }^{20-22}$ Activation of TLR4 by LPS requires a variety of downstream adapters, such as myeloid differentiation primary response protein 88 (MyD88), TIRdomain-containing adaptor-inducing interferon- $\beta$ (TRIF) and TRIF-related adaptor molecule (TRAM), which are essential for signaling the receptor. ${ }^{23,24}$ The recruitment of these adapters may further activate downstream pathways which culminate in the activation of transcription factors that, in effect, induce a plethora of pro-inflammatory genes. ${ }^{24-26}$ In additions, the gut dysbiosis has the impacts to the brain work for instance consideration, focus, intellectual capacity and longing for. Consequently, this research intends to locate the instructive correspondence between the synbiotic supplement and gut-brain axis in cigarette smokers.

\section{MATERIALS AND METHODS}

In this research, we considered the impacts of synbiotic supplement on gut-brain axis in the cigarette smokers. The study was affirmed by and performed under the guidelines of the Research Ethics Committee of Mae Fah Luang University, Thailand.

\section{Participants}

Populations in this exploration were the smokers aged 18-70 years of age who lived in Mahasarakham province with no history of cerebrovascular diseases, brain or abdominal surgery, taking hormones or medications affected brainwaves. Our samples were 18-70 years of age male or female smokers lived in Mahasarakham province coordinated the inclusion criteria, which were somewhere in the range of one and five years of cigarette smoking, score $>/=$ four of Fagerstrom Test for Nicotine Dependence, no history of cerebrovascular diseases, brain or abdominal surgery, taking hormones or medications affected brainwaves, right-handed prevailing, typical vision and hearing, no utilization of antibiotic agents inside about fourteen days prior, no history of colon purifying treatment inside about fourteen days prior, no history of anti-inflammatory drugs inside about fourteen days prior, 
no pregnancy and lactation. We examined in one gathering contained 20 participants.

\section{Synbiotic supplement}

Synbiotics were manufactured by Lactomason Korea Co., Ltd. and contained Lactobacillus rhamnosus, Lactobacillus paracasei, Lactobacillus reuteri, Lactobacillus salivarius, Bifidobacterium lactis, Bifidobacterium breve, Bifidobacterium longum. Total of probiotics contained $25 \times 10^{9}$ colony forming unit per grams as well as included Inulin 4 grams, Fructooligosaccharide 2 grams and Galactooligosaccharide 2 grams. Sachet was in aluminium foil.

\section{Intervention}

Firstly, we reached participants to make an arrangement in the first part of the day. At the arrangement, we clarified the targets, technique, advantages and reactions of the examination in subtleties. After that they marked deliberately the educated assent regarding the exploration. We gave them questionnaires about the individual data, Fagerstrom Test for Nicotine Dependence and Craving Score. At that point we took the primary stool assessment for real-time PCR for Staphylococcus aureus, Bifidobacterium spp., Lactobacillus spp., Enterobacteriaceae, Bacteroides spp., Firmicutes, Clostridium spp., Gram positive bacteria, Gram negative bacteria, and Total bacteria to record the measure of intestinal microbiome. This was for microbiology. In natural chemistry, we gathered the primary blood test for hsCRP, ESR, IL-1b, IL-6, TNF $\alpha$, LPS, the monocyte count to high-density lipoprotein cholesterol proportion (MHR) to record level of inflammatory markers. We additionally led Lactulose-Mannitol test, gathered urine for Lactulose and Mannitol test before taking the sugar and after for six hours. Finally, we checked physiology of the brain to assess cognition by estimating brainwaves, event-related potentials, and developing neuroimaging, which may take around 60 minutes. At last, we gave them synbiotic supplement to allow one sachet with meal in the day for about fourteen days. We made a meeting with the subjects to get the following synbiotic supplement at regular intervals for two months. Following two months, we did nearly everything equivalent to the main visit, which were noting the second Fagerstrom Test for Nicotine Dependence, Craving Score, the second stool assessment for real-time PCR for Staphylococcus aureus, Bifidobacterium spp., Lactobacillus spp., Enterobacteriaceae, Bacteroides spp., Firmicutes, Clostridium spp., Gram positive bacteria, Gram negative bacteria, and Total bacteria, the subsequent blood test for hsCRP, ESR, IL-1b, IL-6, TNF $\alpha$, LPS, MHR, urine test for Lactulose-Mannitol test.

\section{Data analysis}

All of the results were evaluated and analyzed by descriptive analysis, paired t-test or Wilcoxon signed rank test, and
Pearson or Spearman Rank correlation for measuring the strength of their association. A p-value lower than 0.05 was considered significant.

\section{RESULTS}

\section{A. Demographic information of the participants}

Two participants were dropped out, bringing about eighteen subjects took an interest in this research. The demographic data were appeared in Table 1. All members were seventeen males and one female, as $94.44 \%$ and $5.56 \%$ of all participants separately. There was just a single individual matured under twenty years of age, as about $5.5 \%$. Five members matured 20-39 years of age and ten members matured $40-59$ years old were about $27.8 \%$ and $55.6 \%$ of all participants separately. Two participants matured 60-and-above year old took an interest in the measure of $11.1 \%$ of the considerable number of members. Mean and variance difference of time of them were 42.39 and 12.71 years separately. Of the considerable number of participants, seven were single and eleven were married.

\section{B. Fagerstrom test for nicotine dependence}

In Fagerstrom Test for Nicotine Dependence, mean and variance of pre-test were 8.1765 and 0.1544 , while mean and variance of post-test were 7.7059 and 0.5956 separately. $P$-value was 0.0156 . These were appeared in Table 2.

\section{Craving for smoke}

Where as the results of craving smoke in the pre-test were 56 in mean and 27.6250 in variance, the consequences of desiring for smoke in the post-test were 50.8824 in mean and 25.4853 in variance. Table 3 demonstrates the after effects of craving for smoke.

\section{Plasma LPS level}

The mean plasma LPS rate was $23.405 \mathrm{mg} / \mathrm{dL}$ with a difference of $8.31 \mathrm{mg} / \mathrm{dL}$ prior to the ingestion of the synbiotics. After two months, mean with variance of

\begin{tabular}{|c|c|c|c|}
\hline Characteristics & $\mathbf{N}$ & & $\%$ \\
\hline \multicolumn{4}{|l|}{ Gender } \\
\hline Male & 17 & & 94.44 \\
\hline Female & 1 & & 5.56 \\
\hline \multicolumn{4}{|l|}{ Age } \\
\hline Under 20 & 1 & & 5.5 \\
\hline $20-39$ & 5 & & 27.8 \\
\hline $40-59$ & 10 & & 55.6 \\
\hline 60 and above & 2 & & 11.1 \\
\hline Mean & & 42.39 & \\
\hline Variance & & 12.71 & \\
\hline \multicolumn{4}{|l|}{ Status } \\
\hline Single & 7 & & 38.9 \\
\hline Married & 11 & & 61.1 \\
\hline
\end{tabular}




$\begin{aligned} & \text { Table 2: Results of Fagerstrom Test for Nicotine } \\
& \text { Dependence }\end{aligned}$
\begin{tabular}{lccc} 
& \multicolumn{3}{c}{$\mathbf{N}=\mathbf{1 8}$} \\
\cline { 2 - 4 } & Pre-test & & Post-test \\
\hline Mean & 8.1765 & & 7.7059 \\
Variance & 0.1544 & & 0.5956 \\
$P$-value & & 0.0156 & \\
\hline
\end{tabular}

\begin{tabular}{|c|c|c|}
\hline & \multicolumn{2}{|c|}{$\mathrm{N}=18$} \\
\hline & Pre-test & Post-test \\
\hline Mean & 56 & 50.8824 \\
\hline Variance & 27.6250 & 25.4853 \\
\hline$P$-value & & \\
\hline
\end{tabular}

\begin{tabular}{|c|c|c|}
\hline & \multicolumn{2}{|c|}{$\mathrm{N}=18$} \\
\hline & Pre-test (mg/mL) & Post-test (mg/mL) \\
\hline Mean & 23.405 & 17.658 \\
\hline Variance & 8.31 & 7.13 \\
\hline$P$-value & \multicolumn{2}{|c|}{$<0.05$} \\
\hline
\end{tabular}

$7.13 \mathrm{mg} / \mathrm{dL}$ decreased significantly to $17.658 \mathrm{mg} / \mathrm{dL}$. The findings of plasma LPS were shown in Table 4.

\section{DISCUSSION}

This research had the reason to consider about the impact of synbiotic supplement on gut-brain axis in Thai smokers through gut-related biochemical parameters and cognition. The fundamental outcomes have demonstrated that synbiotics influenced nicotine dependence in the Fagerstrom Test. The scores of Fagerstrom Test diminished after admission of synbiotics for about two months altogether. Contrasting with the review of synbiotics and Fagerstrom Test for Nicotine Dependence, no study has been referenced previously.

However, numerous researches have demonstrated positive synergistic impacts for synbiotics on corpulence, diabetes, non-alcoholic fatty liver disease, necrotizing enterocolitis in low birth weight infants, and in the treatment of hepatic encephalopathy. ${ }^{27-31}$ The utilization of probiotic-containing yogurt improved the disposition of those whose state of mind was at first poor. ${ }^{32}$ Our preliminary study has likewise demonstrated that the scores of craving for smoke diminished after synbiotic utilization for about two months altogether. In plasma LPS aspects, data have recently shown that prebiotic-treated mice displayed lower plasma LPS and cytokines and decreased hepatic expression of inflammatory and oxidative stress markers. A reduced inflammatory tone was associated with lower intestinal permeability and increased tight junction integrity. ${ }^{33}$ There were also human studies considering prebiotics and inflammatory or oxidative stress markers. One of them was conducted in participants included 49 diabetic females. They were divided into an intervention group $(n=24)$ as well as a control group $(\mathrm{n}=25)$ and received $10 \mathrm{~g} / \mathrm{d}$ inulin or maltodextrin for 8 weeks, respectively. Inulin-supplemented patients exhibited a significant decrease in FBS $(8.5 \%)$, HbA1c (10.4\%), fasting insulin (34.3\%), homeostasis model assessment of insulin resistance (HOMA-IR) (39.5\%), hs-CRP (35.6\%), TNF- $\alpha$ (23.1\%), and LPS (27.9\%) compared with the maltodextrin group $(\mathrm{p}<0.05)$. Increase in IL-10 was not significant in inulin compared with the maltodextrin group..$^{34}$ As described above, our preliminary outcome also reported a significant decrease in plasma LPS after two months of synbiotic supplementation.

In spite of the fact that these primer discoveries represent to a generally data, it may not reflect every one of the impacts of synbiotic supplement on gut-brain axis in Thai smokers. There was additionally restriction in sample size and short term of the investigation. No placebo control was led in this study. In additions, the participants were huge age contrasts, which were 12.71 years. Therefore, it needs further research to be done in the larger extended way.

\section{CONCLUSION}

The present outcomes indicated essentially decline in the scores of Fagerstrom Test for Nicotine Dependence and also the scores of craving for smoke after intervention. The lipopolysaccharide (LPS) in plasma significantly decreased after the intervention. However, further researches are obligatory to lead in finding a propensity to improve in gut-related biochemical parameter and cognition after admission of synbiotic supplement.

\section{ACKNOWLEDGEMENT}

We thank all of the participants who involved in this study as main data provider.The authors would like to acknowledge the National Research Council of Thailand (Grant No. 126/2561); Postgraduate Studies of Mae Fah Luang University; School of Anti-Aging and Regenerative Medicine, Mae Fah Luang University for the supports, and acknowledge the Innovation Center for Holistic Health, Nutraceuticals and Cosmeceuticals, Faculty of Pharmacy, Chiang Mai University, Thailand, for the necessary provision. Authors also gratefully acknowledge Brain Science and Engineering Innovation Research Group, Mae Fah Luang University grant (2019) (MFU-grant no. 611U109005) and 2020, Thailand for the support. 


\section{REFERENCES}

1. World health organization, WHO Report on the Global Tobacco Epidemic, 2015: Raising Taxes on Tobacco. WHO Report on the Global Tobacco Epidemic World Health Organization, Luxembourg, 2015; 11-198.

2. National Statistical Office Thailand, The survey in smoking and alcohol drinking behavior of Thai population in 2014, 2015.

3. Hoffmann D and Hoffmann I. Letters to the editor, tobacco smoke components. Beitr Tabaksforsch Int 1998; 18: 49-52.

4. Thielen A, Klus $\mathrm{H}$ and Muller L. Tobacco smoke: unraveling a controversial subject. Exp Toxicol Pathol 2008; 60: 141-156.

5. Borgerding $\mathrm{M}$ and Klus $\mathrm{H}$. Analysis of complex mixturescigarette smoke. Exp Toxicol Pathol 2005; 57: 43-73.

6. American Psychiatric Association, Diagnostic and Statistical Manual of Mental Disorders. (5 ${ }^{\text {th }}$ ed.). Washington, DC, 2013.

7. Ussher M, Beard E, Abikoye G, Hajek P and West R. Urge to smoke over 52 weeks of abstinence. Psychopharmacology 2013; 226(1): 83-89.

8. Killen JD and Fortmann SP. Craving is associated with smoking relapse: findings from three prospective studies. Exp Clin Psychopharmacol 1997; 5(2): 137-142.

9. Robinson TE and Berridge KC. The neural basis of drug craving: an incentive-sensitization theory of addiction. Brain Res Brain Res Rev 1993; 18(3): 247-291.

10. Tiffany ST and Conklin CA. A cognitive processing model of alcohol craving and compulsive alcohol use. Addiction 2000; 95(Suppl 2): 145-53.

11. Benjamin JL, Hedin CR, Koutsoumpas A, Ng SC, McCarthy NE, Prescott NJ, et al. Smokers with active Crohn's disease have a clinically relevant dysbiosis of the gastrointestinal microbiota. Inflamm Bowel Dis 2012; 18: 1092-1100.

12. Biedermann L, Zeitz J, Mwinyi J, Sutter-Minder E, Rehman A, Ott SJ, et al. Smoking cessation induces profound changes in the composition of the intestinal microbiota in humans. PLoS One 2013; 8(3): e59260.

13. Biedermann L, Brülisauer K, Zeitz J, Frei P, Scharl M, Vavricka SR, et al. Smoking cessation alters intestinal microbiota: insights from quantitative investigations on human fecal samples using FISH. Inflamm Bowel Dis 2014; 20: 1496-1501.

14. Kobayashi T and Fujiwara K. Identification of Heavy Smokers through Their Intestinal Microbiota by Data Mining Analysis. Biosci Microbiota Food Heal 2013; 32: 77-80.

15. Kobayashi $\mathrm{T}$ and Fujiwara K. Comparison of the accuracy and mechanism of data mining identification of the intestinal microbiota with 7 restriction enzymes. Biosci Microbiota Food Heal 2013; 32: 139-148.

16. Sapkota AR, Berger $S$ and Vogel TM. Human pathogens abundant in the bacterial metagenome of cigarettes. Environ Health Perspect 2010; 118: 351-356.

17. Rogers MA, Greene TM, Saint S, Chenoweth CE, Malani PN, Trivedi I and Aronoff DM. Higher rates of Clostridium difficile infection among smokers. PLoS One 2012; 7: e42091.

18. Gourbeyre P, Denery S and Bodinier M. Probiotics, prebiotics, and synbiotics: Impact on the gut immune system and allergic reactions. J Leukoc Biol 2011; 89: 685-695.

19. Pandey KR, Naik SR and Babu VV. Probiotics, prebiotics and synbiotics—A review. J Food Sci Technol 2015; 52: 7577-7587.

20. Boonen B, Alpizar YA, Sanchez A, Lopez-Requena A, Voets T and Talavera K. Differential effects of lipopolysaccharide on mouse sensory TRP channels. Cell Calcium 2018; 73: 72-81.

21. Alpizar YA, Boonen B, Sanchez A, Jung C, Lopez-Requena A, Naert $R$, et al. TRPV4 activation triggers protective responses to bacterial lipopolysaccharides in airway epithelial cells. Nat Commun 2017; 8: 1059.

22. Meseguer V, Alpizar YA, Luis E, Tajada S, Denlinger B, Fajardo $O$, et al. TRPA1 channels mediate acute neurogenic inflammation and pain produced by bacterial endotoxins. Nat Commun 2014; 5: 3125.

23. Fitzgerald KA, McWhirter SM, Faia KL, Rowe DC, Latz E, Golenbock DT, et al. IKKepsilon and TBK1 are essential components of the IRF3 signaling pathway. Nat Immunol 2003; 4: 491-496.

24. Ruckdeschel K, Pfaffinger G, Haase R, Sing A, Weighardt H, Hacker G, et al. Signaling of apoptosis through TLRs critically involves toll/IL-1 receptor domain-containing adapter inducing IFN-beta, but not MyD88, in bacteria-infected murine macrophages. J Immunol 2004; 173: 3320-3328.

25. Zughaier SM, Zimmer SM, Datta A, Carlson RW and Stephens DS. Differential induction of the toll-like receptor 4-MyD88-dependent and -independent signaling pathways by endotoxins. Infect Immun 2005; 73: 2940-2950.

26. Gray P, Dagvadorj J, Michelsen KS, Brikos C, Rentsendorj A, Town $T$, et al. Myeloid differentiation factor-2 interacts with Lyn kinase and is tyrosine phosphorylated following lipopolysaccharide-induced activation of the TLR4 signaling pathway. J Immunol 2011; 187: 4331-4337.

27. Islek A, Sayar E, Yilmaz A, Baysan BO, Mutlu D and Artan R. The role of bifidobacterium lactis b94 plus inulin in the treatment of acute infectious diarrhea in children. Turk $\mathrm{J}$ Gastroenterol 2014; 25: 628-633.

28. Asemi Z, Khorrami-Rad A, Alizadeh SA, Shakeri H and Esmaillzadeh A. Effects of synbiotic food consumption on metabolic status of diabetic patients: A double-blind randomized cross-over controlled clinical trial. Clin Nutr 2014; 33: 198-203.

29. Steed H, Macfarlane GT, Blackett KL, Bahrami B, Reynolds N, Walsh SV, et al. Clinical trial: The microbiological and immunological effects of synbiotic consumption-A randomized double-blind placebo-controlled study in active crohn's disease. Aliment Pharmacol Ther 2010; 32: 872-883.

30. Dilli D, Aydin B, Fettah ND, Ozyazici E, Beken S, Zenciroglu A, et al. The propre-save study: Effects of probiotics and prebiotics alone or combined on necrotizing enterocolitis in very low birth weight infants. J Pediatr 2015; 166: 545-551.

31. Eslamparast $T$, Poustchi $H$, Zamani $F$, Sharafkhah $M$, Malekzadeh R and Hekmatdoost A. Synbiotic supplementation in nonalcoholic fatty liver disease: A randomized, double-blind, placebo-controlled pilot study. Am J Clin Nutr 2014; 99: 535-542.

32. Benton D, Williams $\mathrm{C}$ and Brown A. Impact of consuming milk drink containing a probiotic on mood and cognition. European $\mathrm{J}$ Clinical Nutrition 2007; 61: 355-361.

33. Cani PD, Possemiers S, Van de Wiele T, Guiot Y, Everard A, Rottier $\mathrm{O}$, et al. Changes in gut microbiota control inflammation in obese mice through a mechanism involving GLP-2-driven improvement of gut permeability. Gut 2009; 58(8): 1091-1103.

34. Dehghan P, Gargari BP, Jafar-Abadi MA and Aliasgharzadeh A. Inulin controls inflammation and metabolic endotoxemia in women with type 2 diabetes mellitus: a randomized-controlled clinical trial. Int J Food Sci Nutr 1024;65:117-123. 


\section{Authors Contribution:}

PP-Concept and design of the study, manuscript preparation, statistically analyzed; VS- Concept and design of the study, statistically analyzed and interpreted; PP- Concept and design of the study, statistically analyzed and interpreted; TN- Concept and design of the study, critical revision of the manuscript; CC-Concept and design of the study, critical revision of the manuscript; SS- Critical revision of the manuscript; PS- Concept and design of the study, manuscript preparation, critical revision of the manuscript.

\section{Work attributed to:}

Brain Science and Engineering Innovation Research Group, School of Anti-Aging and Regenerative Medicine, Mae Fah Luang University, Thailand. Innovation Center for Holistic Health, Nutraceuticals and Cosmeceuticals, Faculty of Pharmacy, Chiang Mai University, Thailand;

This research paper is a part of first author's Dissertation in Anti-Aging and Regenerative Medicine, School of Anti-Aging and Regeneration Medicine, Mae Fah Luang University, Bangkok, Thailand.

\section{Orcid ID:}

Dr. Parama Pratummas- (i) https://orcid.org/0000-0002-1472-3803

Dr. Vachrintr Sirisapsombat- https://orcid.org/0000-0003-3575-6759

Dr. Phuttharaksa Phumcharoen- (1) https://orcid.org/0000-0002-4438-9172

Professor Dr. Thamthiwat Nararatwanchai- (i) https://orcid.org/0000-0002-4189-8115

Dr. Chaiyavat Chaiyasut- (1) https://orcid.org/0000-0002-1633-2419

Dr. Sasithorn Sirilun- (1) https://orcid.org/0000-0002-1508-2358

Dr. Phakkharawat Sittiprapaporn- (iD https://orcid.org/0000-0002-4103-9396

Source of Support: National Research Council of Thailand (Grant No. 126/2561); Postgraduate Studies of Mae Fah Luang University; School of Anti-Aging and Regenerative Medicine, Mae Fah Luang University, Thailand; Innovation Center for Holistic Health, Nutraceuticals and Cosmeceuticals, Faculty of Pharmacy, Chiang Mai University, Thailand; Brain Science and Engineering Innovation Research Group, Mae Fah Luang University grant (2019) (MFU-grant no. 611U109005) and 2020, Thailand, Conflict of Interest: None. 